“C 2011 IEEE. Personal use of this material is permitted. Permission from IEEE must be obtained for all other uses, in any current or future media, including reprinting/republishing this material for advertising or promotional purposes, creating new collective works, for resale or redistribution to servers or lists, or reuse of any copyrighted component of this work in other works." 


\title{
Development of a Bayesian Recursive Algorithm to Find Free-spaces for an Intelligent Wheelchair
}

\author{
Anh V. Nguyen, Steven Su, Member, IEEE, and Hung T. Nguyen, Senior Member, IEEE
}

\begin{abstract}
This paper introduces a new shared control strategy for an intelligent wheelchair using a Bayesian recursive algorithm. Using the local environment information gathered by a laser range finder sensor and commands acquired through a user interface, a Bayesian recursive algorithm has been developed to find the most appropriate free-space, which corresponds to the highest posterior probability value. Then, an autonomous navigation algorithm will assist to manoeuvre the wheelchair in the chosen freespace. Experiment results demonstrate that the new method provides excellent performance with great flexibility and fast response.
\end{abstract}

\section{INTRODUCTION}

$\mathrm{F}$ OR people with disabilities as well as elderly people, wheelchairs have played a very important role which can significantly improve their quality of life. With the assistance of wheelchairs, these people are capable to move around their home, go to work or do their daily work independently. However, while a majority of users control their electric power wheelchairs efficiently, there are also many people who find it difficult or impossible to drive normal electric wheelchairs [1]. With the advent of new technology, power wheelchairs with significant innovation, called intelligent wheelchairs, have been developed to meet demands of this population.

Generally, an intelligent wheelchair has an onboard personal computer that allows it to interface with external devices and to implement safe navigation algorithms to bring the user to her/his desired goals. Unlike an autonomous robot which has the ability to navigate by itself, an intelligent wheelchair always has two control sources: the computer and the user. Approaches that integrate the human inputs with autonomous behaviors vary from fully autonomous to fully cooperative.

In the fully autonomous mode, the intelligent wheelchair operates in a manner very similar to autonomous robots [2]. However, the main disadvantage of the full autonomous mode is that the user is not involved in the navigating process. Conversely, in a fully cooperative

Anh V. Nguyen is with Faculty of Engineering and Information Technology, University of Technology, Sydney, Broadway, NSW 2007, Australia, (e-mail: Anh.Nguyen-3@student.uts.edu.au ).

Steven Su is with Faculty of Engineering and Information Technology, University of Technology, Sydney, Broadway, NSW 2007, Australia, (email: Steven.Su@uts.edu.au ).

Hung T. Nguyen is with Faculty of Engineering and Information Technology, University of Technology, Sydney, Broadway, NSW 2007, Australia, (e-mail: Hung.Nguyen@uts.edu.au ). mode, the user is considered as a partner of the wheelchair [3], allowing him/her to cooperate in all levels through very complex preset commands.

Another mode, which can be considered as an alternative of the two above-mentioned categories, relates to shared control systems. In this mode, both the user and autonomous commands are combined concurrently to perform navigating tasks.

There are methods that base on the user's ability to decide the level of combination between the user and autonomous commands. Some simply estimate the consistency of the user inputs [4]. Others measure the user's driving skills or their health conditions, and then decide at which level the autonomous system needs to assist users $[5,6]$. These approaches have difficulties to indentify the user's intention, and they are usually applicable for only a specific individual.

Other shared control methods are based on the consideration of both environment information and the user's commands concurrently. For example, the information from environment and user commands are used to activate the appropriate available autonomous tasks [7]. In a recent study, a method of minimizing a function $\mathrm{C}_{\text {share }}$ based on combining both user's command and nominated free-spaces to find the proper navigation direction [8]. In [9], a Bayesian technique was used to choose appropriate existing autonomous tasks. Both methods have been demonstrated to work well in various dynamic environments.

In this paper, we develop a new shared control method in which a Bayesian recursive algorithm will be applied to determine the most appropriate free-space based on information from the user's commands and environment measurement. The paper is organized as follows. The wheelchair instrumentation will be discussed in Section II, and, a Bayesian recursive algorithm is introduced in Section III. Experimental results will be shown in Section IV. The last section will present our conclusion.

\section{WHEELCHAIR INSTRUMENTATION}

The hardware overview of our intelligent wheelchair based on a commercial powered wheelchair is shown in Fig. 1. This wheelchair has been modified to include three additional items: a) Mac mini computer with $2.66 \mathrm{GHz}$ Core 2 Duo Processor, b) user interface, and c) measurement sensors. With this modification, the wheelchair allows the user to operate in both manual mode 
and assistive mode. By choosing a mode on the master control panel, the user can use the joystick to drive the wheelchair manually or drive the wheelchair with the assistance from semi-autonomous navigation software in the assistive mode.

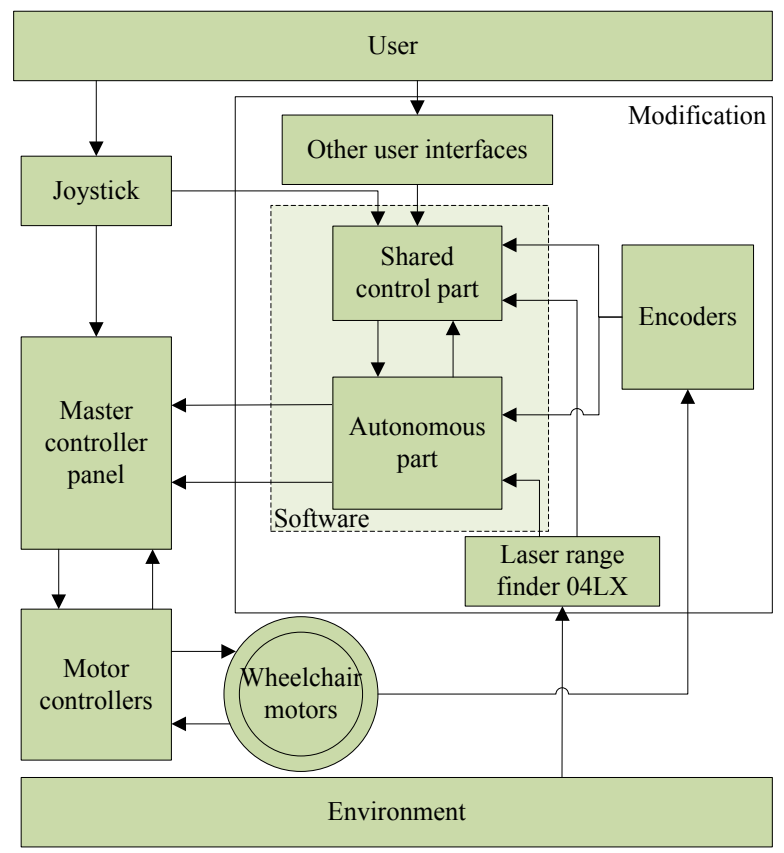

Fig.1. The wheelchair Hardware Overview

User interfaces: Our system has been designed to work with both continuous and discrete or sparse commands from a user. Through recent years, we have been developing a number of novel user interfaces, which facilitate the disabled people, including a brain-computer interface (BCI), a head-movement system or a control system through iPhone $4 / \mathrm{iPad}$.

Measurement sensors: The wheelchair is equipped with sensors to obtain information about local environment. In addition to the wheel encoders to measure the position of the wheelchair, a camera CCD, a laser range finder URG04LX or an ultrasonic range finder SRF01 can be used to measure the distances of surrounding obstacles. The URG04LX which can provide accurate range data and highresolution high speed streaming data will be used in this study.

Along with novel ad-on devices, a number of different assistive navigation algorithms have been developed for this wheelchair [8-11]. Its system, considered as a semiautonomous system, mainly consists of a shared control part and an autonomous part.

Shared control part: This part plays a role in combining the user command and environment information to find an appropriate navigation direction for the wheelchair. To do this, we propose a new algorithm based on a Bayesian recursive estimation framework.

Autonomous part: In this part, we use a Bayesian neural network as developed in [10] to generate control signals to drive the wheelchair corresponding to information from the shared control part and environment measurement.

Our integrated program for the wheelchair is implemented by using the multi-threading approach in Labwindows/CVI 8.5 software, a product of National Instruments, based on Microsoft windows XP Service Pack 3 (SP3).

\section{SHARED CONTROL FRAMEWORK FOR THE WHEELCHAIR}

In order to make decisions based on the surrounding environment and the user's demand, the intelligent wheelchair will use external devices to understand the environment and to send information to its controller. In general, the signals obtained from these external devices are noisy and limited. For example, the information relating to the distance of obstacles received from a laser range finder URG-04LX depicts only the scanning plane, and not the overall environment. In addition, as people with disabilities often give inconsistent commands, the repeatability of their intention could be uncertain.

To cope with changes of environment and uncertain signals, a Bayesian recursive estimation algorithm based on probabilistic information is considered as an attractive option because it is well-suited for dealing with insufficient information.

Assuming that during the navigating process, at the time $\mathrm{t}$, a user wants to move to a free-space which may be in front $\left(x_{t}=F\right)$, on the left side $\left(x_{t}=L\right)$, or on the right side $\left(x_{t}=R\right)$. By modeling both environment and control information, a Bayesian recursive estimation algorithm can find the most suitable free-space $x_{t}$ which has the highest posterior probability value at time $t$.

This algorithm is recursively applied, that is, the posterior probability of free-space $P\left(x_{t}\right)$ at the time t is determined by considering 3 factors: the posterior probability $P\left(x_{t-1}\right)$ at time $\mathrm{t}-1$, the most recent control command $U_{t}$ and the latest environment measurement $Z_{t}$.

Assuming that at the time $t$, the wheelchair controller has received a stream of information

$$
I=\left\{U_{1}, Z_{1}, U_{2}, Z_{2}, \ldots, U_{t}, Z_{t}\right\}
$$

We denote that $Z_{1: t}$ and $U_{1: t}$ are the set of all measurement and control data acquired from the beginning to the time $t$.

In order to find the posterior probability at time $\mathrm{t}$ of a free-space $x_{t}$, first, the prior probability $\bar{P}\left(x_{t}\right)$ needs to be found as follows

$$
\begin{aligned}
\bar{P}\left(x_{t}\right) & =P\left(x_{t} \mid Z_{1: t-1}, U_{t}\right) \\
& =\int P\left(x_{t} \mid x_{t-1}, Z_{1: t-1}, U_{t}\right) P\left(x_{t-1} \mid Z_{1: t-1}, U_{1: t}\right) d x_{t-1}
\end{aligned}
$$

As there are three free-space types, and presenting the equation (1) in a discrete form, we have

$$
\bar{P}\left(x_{t}\right)=\sum_{j=F, L, R} P\left(x_{t} \mid x_{t-1}^{j}, Z_{1: i-1}, U_{t}\right) P\left(x_{t-1}^{j} \mid Z_{1: i-1}, U_{1: t}\right)
$$

Next, the posterior probability can be calculated based on the prior probability 


$$
P\left(x_{t}\right)=P\left(x_{t} \mid Z_{1: t}, U_{1: t}\right)=\eta P\left(Z_{t} \mid x_{t}\right) \bar{P}\left(x_{t}\right)
$$

Where $\eta$ is a constant and calculated by

$$
\eta=1 / \sum_{j=F, L, R} P\left(Z_{t} \mid x_{t}^{j}, Z_{1: t-1}, U_{1: t}\right) P\left(x_{t}^{j} \mid Z_{1: t-1}, U_{1: t}\right)
$$

The program structure to implement this algorithm is shown as Fig. 2. After initializing the parameters, the prior probability is determined by Eq. (2). Then, the posterior probability is calculated by Eq. (3). Finally, the most suitable free-space will be found.

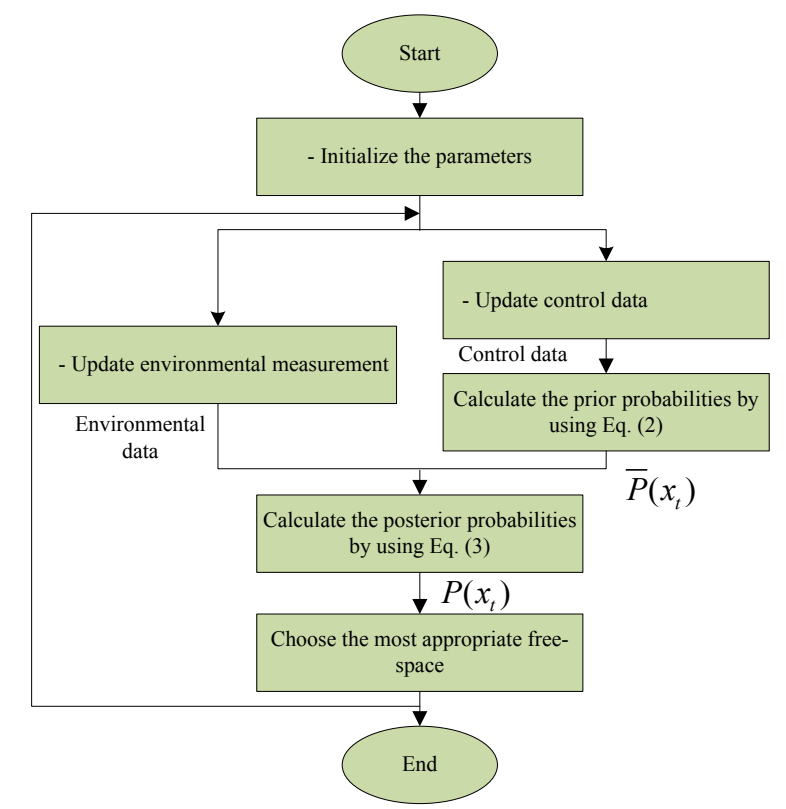

Fig.2. The shared control framework using Bayesian Recursive algorithm

After the free-space is found, the information will be passed to the autonomous part. As mentioned above, a trained neural network is used for this part. Inputs of the neural network are data received from the laser range finder and a navigation direction, and its outputs are the parameters to control the speed and steering. In a previous study from our group [8], the navigation direction was defined as a direction to a chosen free-space. In this study, the navigation direction is calculated by considering the wheelchair's current position and the free-space.

\section{EXPERIMENT RESULTS}

The experiments were designed to test the performance of the new method. In these experiments, data was recorded at 100 millisecond intervals. Free-spaces are determined based on the procedure described in [10].

\section{A. Initialization}

In order to implement the Bayesian recursive estimation algorithm, the parameters are initialized at the beginning. At time $\mathrm{t}=0$, the prior probabilities are set equally as follows.

$$
P\left(x_{0}=F\right)=P\left(x_{0}=L\right)=P\left(x_{0}=R\right)=1 / 3
$$

The conditional probabilities corresponding to the freespace measurement and user's commands are set as in table

\begin{tabular}{|c|c|c|c|c|c|c|c|c|}
\hline \multicolumn{3}{|c|}{ In front } & \multicolumn{3}{|c|}{ On the left } & \multicolumn{3}{|c|}{ On the right } \\
\hline $\bar{Z}_{t} x_{t}$ & $\mathrm{~F}$ & $\bar{F}$ & $\mathrm{Z}_{\mathrm{t}} \quad \mathrm{X}_{\mathrm{t}}$ & $\mathrm{L}$ & $\bar{L}$ & $\mathrm{Z}_{\mathrm{t}} \mathrm{X}_{\mathrm{t}}$ & $\mathrm{R}$ & $\bar{R}$ \\
\hline$F$ & 0.8 & 0.2 & $\mathrm{~L}$ & 0.8 & 0.2 & $\mathrm{R}$ & 0.8 & 0.2 \\
\hline $\bar{F}$ & 0.2 & 0.8 & $\bar{L}$ & 0.2 & 0.8 & $\bar{R}$ & 0.2 & 0.8 \\
\hline
\end{tabular}

I-II.

Table I. Conditional probabilities for a free-space measurement

\begin{tabular}{|c|c|c|c|c|c|c|c|c|c|c|c|}
\hline \multicolumn{4}{|c|}{$\mathrm{U}_{\mathrm{t}}=\mathrm{F}$} & \multicolumn{4}{|c|}{$\mathrm{U}_{\mathrm{t}}=\mathrm{L}$} & \multicolumn{4}{|c|}{$\mathrm{U}_{\mathrm{t}}=\mathrm{R}$} \\
\hline $\mathrm{x}_{\mathrm{t}} \mathrm{x}_{\mathrm{t}-\mathrm{1}}$ & F & $\mathrm{L}$ & $\mathrm{R}$ & $x_{t} x_{t-1}$ & $\mathrm{~F}$ & $\mathrm{~L}$ & $\mathrm{R}$ & $\mathrm{x}_{\mathrm{t}} \quad \mathrm{x}_{\mathrm{t}-1}$ & $\mathrm{~F}$ & $\mathrm{~L}$ & $\mathrm{R}$ \\
\hline $\mathrm{F}$ & 0.8 & 0.5 & 0.5 & $\mathrm{~F}$ & 0.3 & 0.4 & 0.2 & $\mathrm{~F}$ & 0.3 & 0.5 & 0.4 \\
\hline $\mathrm{L}$ & 0.1 & 0.4 & 0.1 & L & 0.6 & 0.6 & 0.5 & $\mathrm{~L}$ & $\begin{array}{ll}0.1 \\
\end{array}$ & 0.2 & 0 \\
\hline $\mathrm{R}$ & 0.1 & \begin{tabular}{|l|}
0.1 \\
\end{tabular} & 0.4 & $\mathrm{R}$ & 0.1 & 0 & 0.3 & $\mathrm{R}$ & 0.6 & \begin{tabular}{|l|}
0.3 \\
\end{tabular} & 0.6 \\
\hline
\end{tabular}

Table II. Conditional probabilities for a user's command

\section{B. Performance results}

After initializing the relevant parameters, we test wheelchair's performance in the environment with various free-spaces as in Fig. 3.

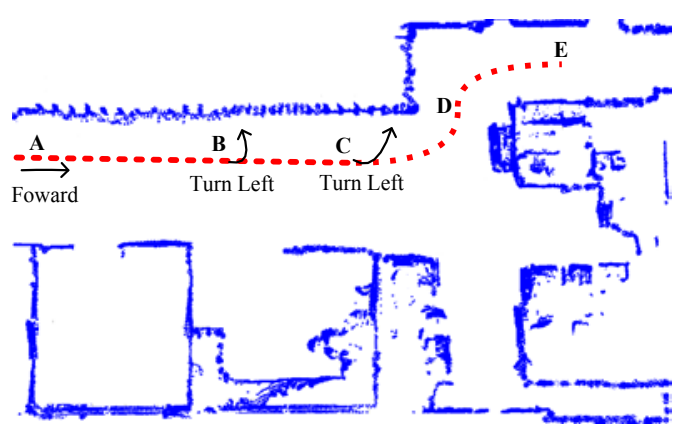

Fig.3. Wheelchair's trajectory when using Bayesian recursive algorithm

At point A, the wheelchair goes forward with the smooth and optimal trajectory as the user points to forward and in front free-space is detected by a laser range finder.

At point $\mathrm{B}$, when the user tests the system behavior by giving a turn left command although there is a wall in this side, the wheelchair still keeps moving forward consistently. This is explained as following. At time t, the user gives a turn left command, control data are updated $U_{t}=L$ and the environment measurement is $Z_{t}=$ $(F) \&(R)$. The wheelchair controller calculates the posterior probabilities by two consecutive steps.

Step 1: Calculating the prior probabilities by Eq. (2).

$$
\begin{aligned}
& \bar{P}\left(x_{t}=F\right)=\sum_{j=F, L, R} P\left(x_{t}=F \mid x_{t-1}^{j}, U_{t}=L\right) P\left(x_{t-1}^{j}\right)=0.34 \\
& \bar{P}\left(x_{t}=L\right)=\sum_{j=F, L, R} P\left(x_{t}=L \mid x_{t-1}^{j}, U_{t}=L\right) P\left(x_{t-1}^{j}\right)=0.56 \\
& \bar{P}\left(x_{t}=R\right)=\sum_{j=F, L, R} P\left(x_{t}=R \mid x_{t-1}^{j}, U_{t}=L\right) P\left(x_{t-1}^{j}\right)=0.10
\end{aligned}
$$

Step 2: Calculating the posterior probabilities by Eq. (3).

$$
\begin{aligned}
& P\left(x_{t}=F\right)=\frac{P\left(Z_{t}=F \& R \mid x_{t}=F\right) \bar{P}\left(x_{t}=F\right)}{\sum_{j=F, L, R} P\left(Z_{t}=F \& R \mid x_{t}^{j}\right) \bar{P}\left(x_{t}^{j}\right)}=0.68 \\
& P\left(x_{t}=L\right)=\frac{P\left(Z_{t}=F \& R \mid x_{t}=F\right) \bar{P}\left(x_{t}=L\right)}{\sum_{j=F, L, R} P\left(Z_{t}=F \& R \mid x_{t}^{j}\right) \bar{P}\left(x_{t}^{j}\right)}=0.24 \\
& P\left(x_{t}=R\right)=\frac{P\left(Z_{t}=F \& R \mid x_{t}=F\right) \bar{P}\left(x_{t}=R\right)}{\sum_{j=F, L, R} P\left(Z_{t}=F \& R \mid x_{t}^{j}\right) \bar{P}\left(x_{t}^{j}\right)}=0.31
\end{aligned}
$$


From these values, we can see that although prior probability of $x_{t}=L$ is the highest value in the first step, its posterior probability is the smallest value in the second step because no free-space is found in this direction. Thus, the wheelchair keeps moving forward due to the posterior probability of $x_{t}=F$ is the highest.

At point $\mathrm{C}$, after receiving a turn left command, and a left-side free-space is also determined, the wheelchair smoothly turns left into a small free-space.

At point $\mathrm{D}$, the wheelchair automatically turns right to go to $\mathrm{E}$ due to a free-space on right side is detected.

A comparison between the new proposed method and previous study using the shared function $C_{\text {Share }}$ [8] is shown as Fig.4. The wheelchair moves forward quite closely to the wall until point $\mathrm{G}$. At point $\mathrm{G}$, a turn right command is given, the wheelchair with the shared function $C_{\text {Share }}$ determines a navigation direction to the free-space, and starts turning left into the small door immediately. As a result, collision with the door frame is imminent. With the new method, after determining the available free-space, the autonomous part produces a suitable navigation direction. In this situation, by keeping moving ahead slightly before turning right in the optimal trajectory, the wheelchair can safely enter the door.
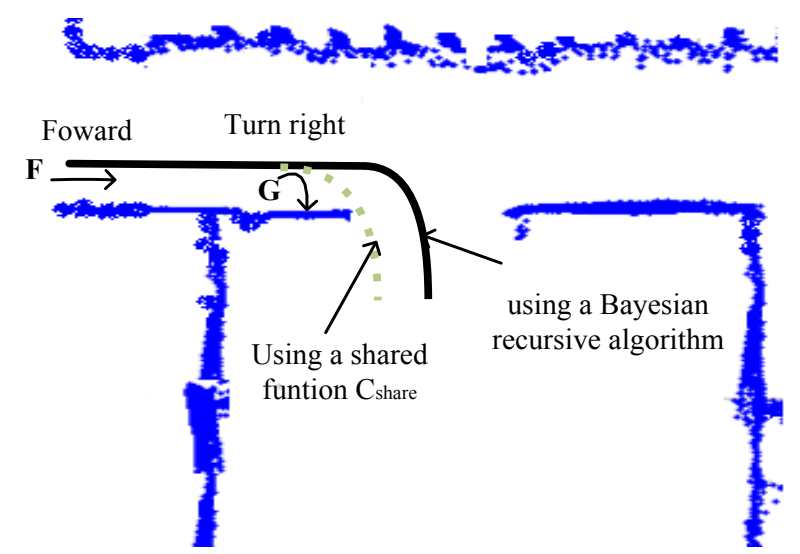

Fig.4. Comparison between the Bayesian recursive algorithm and the shared function $\mathrm{C}_{\text {shared }}$ method

During the experiments, the wheelchair performs very reliably in this dynamic environment even when the maximum speed of the wheelchair is set to $0.8 \mathrm{~m} / \mathrm{s}$. This excellent performance has been obtained due to the reasonable computational burden which is distributed to the synchronous parts.

\section{CONCLUSION}

In this paper, a Bayesian recursive estimation algorithm is implemented to find the most appropriate free-space based on the information from a user's command, measurements from the laser range finder and the wheel encoders. A neural network based on Bayesian framework generates appropriate control signals to drive the wheelchair into the chosen free-space safely with smooth and optimal trajectories. Experimental results on the wheelchair in a real environment have demonstrated the effectiveness of this new method.

Currently, we use only one neural network to generate control signals to drive the wheelchair. Thus, future work will focus on expanding a number of different networks specialized in each situation, and the result of this Bayesian recursive algorithm will play a role to activate the suitable neural network. Also, this technique is compared with only the shared function method until now, therefore a comparison between this method and different algorithms at different conditions will be implemented to have an overall performance comparison.

\section{REFERENCES}

[1] L. Fehr, W. E. Langbein, and S. B. Skaar, "Adequacy of power wheelchair control interfaces for persons with severe disabilities: A clinical survey," Journal of Rehabilitation Research and Development, vol. 37, pp. 353-360, May-Jun 2000.

[2] H. Seki, S. Kobayashi, Y. Kamiya, M. Hikizu, and H. Nomura, "Autonomous/semi-autonomous navigation system of a wheelchair by active ultrasonic beacons," in Robotics and Automation, 2000. Proceedings. ICRA '00. IEEE International Conference on, 2000, pp. 1366-1371 vol.2.

[3] C. Galindo, J. Gonzalez, and J. A. Fernandez-Madrigal, "Control Architecture for Human\&amp;\#8211;Robot Integration: Application to a Robotic Wheelchair," Systems, Man, and Cybernetics, Part B: Cybernetics, IEEE Transactions on, vol. 36, pp. 1053-1067, 2006.

[4] S. P. Parikh, V. Grassi Jr, V. Kumar, and J. Okamoto Jr, "Integrating Human Inputs with Autonomous Behaviors on an Intelligent Wheelchair Platform," Intelligent Systems, IEEE, vol. 22, pp. 33-41, 2007.

C. Urdiales, B. Fernandez-Espejo, R. Annicchiaricco, F. Sandoval, and C. Caltagirone, "Biometrically Modulated Collaborative Control for an Assistive Wheelchair," Neural Systems and Rehabilitation Engineering, IEEE Transactions on, vol. 18, pp. 398-408, 2010.

[6] D. Vanhooydonck, E. Demeester, A. Hüntemann, J. Philips, G. Vanacker, H. Van Brussel, and M. Nuttin, "Adaptable navigational assistance for intelligent wheelchairs by means of an implicit personalized user model," Robotics and Autonomous Systems, vol. 58, pp. 963-977, 2010.

[7] R. C. Simpson and S. P. Levine, "Automatic adaptation in the NavChair Assistive Wheelchair Navigation System," Rehabilitation Engineering, IEEE Transactions on, vol. 7, pp. 452-463, 1999.

[8] H. T. Trieu, H. T. Nguyen, and K. Willey, "Shared Control Strategies for Obstacle Avoidance Tasks in an Intelligent Wheelchair," in 2008 30th Annual International Conference of the Ieee Engineering in Medicine and Biology Society, Vols 18, 2008, pp. 4254-4257.

[9] H. T. Trieu, K. Willey, and H. T. Nguyen, "Adaptive shared control strategies based in the Bayesian recursive technique in an intelligent wheelchair," in Engineering in Medicine and Biology Society, 2009. EMBC 2009. Annual International Conference of the IEEE, 2009, pp. 7118-7121.

[10] H. T. Trieu, H. T. Nguyen, and K. Willey, "Obstacle avoidance for power wheelchair using Bayesian neural network," in Annual International Conference of the Ieee Engineering in Medicine and Biology Society, Vols 1-16, 2007., 2007, pp. 4771-4774.

[11] H. T. Trieu, H. T. Nguyen, and K. Willey, "Advanced Obstacle Avoidance for a Laser Based Wheelchair Using Optimised Bayesian Neural Networks," in 2008 30th Annual International Conference of the Ieee Engineering in Medicine and Biology Society, Vols 1-8, 2008, pp. 3463-3466. 